\title{
Systematic approach to statistics of conductance and shot-noise in chaotic cavities
}

\author{
B. A. Khoruzhenko, ${ }^{1}$ D. V. Savin, ${ }^{2}$ and H.-J. Sommers ${ }^{3}$ \\ ${ }^{1}$ Queen Mary University of London, School of Mathematical Sciences, London E1 4NS, United Kingdom \\ ${ }^{2}$ Department of Mathematical Sciences, Brunel University, Uxbridge UB8 3PH, United Kingdom \\ ${ }^{3}$ Fachbereich Physik, Universität Duisburg-Essen, 47048 Duisburg, Germany \\ (Received 29 May 2009; revised manuscript received 1 August 2009; published 2 September 2009)
}

\begin{abstract}
Applying random matrix theory to quantum transport in chaotic cavities, we develop a powerful method for computing the moments of the conductance and shot-noise (including their joint moments) of arbitrary order and at any number of open channels. Our approach is based on the Selberg integral theory combined with the theory of symmetric functions and is applicable equally well for systems with and without time-reversal symmetry. We also compute higher-order cumulants and perform their detailed analysis. In particular, we establish an explicit form of the leading asymptotic of the cumulants in the limit of the large channel numbers. We derive further a general Pfaffian representation for the corresponding distribution functions. The Edgeworth expansion based on the first four cumulants is found to reproduce fairly accurately the distribution functions in the bulk even for a small number of channels. As the latter increases, the distributions become Gaussian-like in the bulk but are always characterized by a power-law dependence near their edges of support. Such asymptotics are determined exactly up to linear order in distances from the edges, including the corresponding constants.
\end{abstract}

DOI: 10.1103/PhysRevB.80.125301

PACS number(s): 73.23.-b, 73.50.Td, 05.45.Mt, 73.63.Kv

\section{INTRODUCTION}

Quantum transport in mesoscopic systems has been a subject of an intense study during the last decade. ${ }^{1,2}$ Traditionally, the focus of interest has been on statistical properties of the conductance, $g$, and shot-noise, $p$. For noninteracting electrons in a two-terminal conductor at zero temperature, the Landauer-Büttiker scattering formalism ${ }^{3}$ relates these quantities (expressed in their natural units) to the so-called transmission eigenvalues $T_{i}$ of the conductor

$$
g=\sum_{i=1}^{n} T_{i}, \quad p=\sum_{i=1}^{n} T_{i}\left(1-T_{i}\right) .
$$

Here, $n \equiv \min \left(N_{1}, N_{2}\right)$, where $N_{1,2}$ are the number of propagating modes (channels) in the two attached leads. The $T_{i}$ are the eigenvalues of the matrix $t t^{\dagger}$, with $t$ being $N_{1} \times N_{2}$ matrix of transmission amplitudes from the entrance to exit channels. They are mutually correlated random numbers, $0 \leq T_{i}$ $\leq 1$, whose distribution depends on the type of the conductor.

Below, we consider chaotic cavities (open quantum dots). In this case, random matrix theory (RMT) has proved to be successful in describing universal fluctuations in transport through such systems. ${ }^{1,4}$ Within this RMT approach, the joint probability density function $(\mathrm{JPDF}), \mathcal{P}_{\beta}(T)$, of the transmission eigenvalues is induced by the random scattering matrix drawn from one of Dyson's circular ensembles, ${ }^{5}$ according to the global symmetries present in the system. The exact expression for this JPDF is known ${ }^{1,6,7}$ to have the following simple form:

$$
\mathcal{P}_{\beta}(T)=\mathcal{N}_{\beta}^{-1}|\Delta(T)|^{\beta} \prod_{i=1}^{n} T_{i}^{\alpha-1},
$$

where $\alpha=\frac{\beta}{2}\left(\left|N_{1}-N_{2}\right|+1\right)$ and $\Delta(T)=\Pi_{i<j}\left(T_{i}-T_{j}\right)$ denotes the Vandermonde determinant. The Dyson's symmetry index $\beta$ depends on the presence $(\beta=1)$ or absence $(\beta=2)$ of time- reversal symmetry or that of spin-flip symmetry $(\beta=4)$ in the system, thus distinguishing between the three canonical RMT ensembles (orthogonal, unitary, or symplectic, respectively). ${ }^{5}$ The normalization constant $\mathcal{N}_{\beta}$ is given by

$$
\mathcal{N}_{\beta}=\prod_{j=0}^{n-1} \frac{\Gamma\left[1+\frac{\beta}{2}(1+j)\right] \Gamma\left(\alpha+\frac{\beta}{2} j\right) \Gamma\left(1+\frac{\beta}{2} j\right)}{\Gamma\left(1+\frac{\beta}{2}\right) \Gamma\left[1+\alpha+\frac{\beta}{2}(n+j-1)\right]}
$$

and assures that expression (2) is a probability density. It is known generally for discrete positive $n$ and continuous $\alpha$ and $\beta$ as the Selberg integral. ${ }^{5}$

Presently, there is a substantial progress in the understanding of statistics of various transport observables in chaotic cavities that is due to the recent developments of new analytical methods in the theory. Among them, the Selberg integral theory plays a special and important role, see the recent review $^{8}$ for its current status. In the present context, it has been initially applied by two of us ${ }^{9}$ to find the average value of shot-noise and hence the Fano factor exactly. This approach has then been developed further to study full counting statistics of charge transfer ${ }^{10}$ as well as to obtain exact explicit expressions for the shot-noise variance and for the skewness and kurtosis of the charge and conductance distributions $^{11,12}$ (see also Refs. 13-21 for other RMT results on the relevant statistics in chaotic cavities). Since $\beta$ enters the Selberg integral as a continuous parameter, this method allows us to treat all the three ensembles on equal footing, thus giving a powerful alternative to diagrammatic ${ }^{13,18}$ or orthogonal polynomial ${ }^{14,21}$ approaches, especially when the channel numbers are small.

A completely alternative treatment has been recently undertaken within the semiclassical approach ${ }^{22,23}$ which represents quantum transport in terms of classical trajectories connecting the leads. By constructing asymptotic semiclassical 
expansions for transport observables, this approach successfully accounts for both system-specific and universal (RMT) features (see Ref. 24 for a review).

The case of $\beta=2$ (broken time-reversal symmetry) is known for several reasons to be the special one in RMT. For the problem in question, further progress in this case has been made very recently along the following two directions. Novaes ${ }^{25}$ combined the Selberg integral with facts from the theory of symmetric functions to compute nonperturbatively moments of both the transmitted charge and conductance but not those of shot-noise. Alternatively, Osipov and Kanzieper ${ }^{26}$ combined the theory of integrable systems with RMT, as given by Eq. (2), bringing out an effective formalism for calculating the joint cumulants of the conductance and shot-noise. ${ }^{27}$ However, the relevant consideration for the systems with preserved time-reversal symmetry, $\beta$ $=1$, is still lacking.

The distribution functions of the conductance and shotnoise are also studied quite intensively on their own. However, no explicit expressions have been reported so far except for a few cases, namely, for the conductance distribution at $N_{1,2}=1,2$ (Refs. 6, 7, 15, and 28) and for the shot-noise distribution at $N_{1,2}=1 .{ }^{18,29}$ Asymptotic analysis of the both distributions at $N_{1,2} \gg 1$ has been performed very recently in Ref. 30 (see also Ref. 26). To the best of our knowledge, no general results valid at arbitrary $N_{1,2}$ and $\beta \neq 2$ are available thus far. Meanwhile, the conductance distribution with dephasing ${ }^{31}$ has been directly measured in open quantum dots $^{32}$ and in microwave billiards. ${ }^{33}$ The shot-noise power in chaotic cavities has been recently studied experimentally. ${ }^{34}$ Counting electrons in quantum dots is also experimentally accessible. ${ }^{35}$ All this provides an additional motivation for the present study.

In this work we explore further the direction along the lines of Novaes's work ${ }^{25}$ and develop a systematic approach for computing the moments of linear statistics in transmission eigenvalues for the systems with both preserved and broken time-reversal symmetry. This approach yields the moments of the conductance and shot-noise of arbitrary order, including their joint moments and cumulants. In the next section we present the detailed exposition of the method used, including the relevant facts from the theory of symmetric functions. This method is then applied in Sec. III to derive expressions for the moments and cumulants of the conductance and shot-noise in a closed form. Sections IV and V complement this study by investigating the corresponding distribution functions and their asymptotic behavior. Our main findings are summarized and discussed in the concluding Sec. VI.

\section{THE METHOD}

The method is based on expanding powers of the conductance or shot-noise (or any other linear statistic) in Schur functions $s_{\lambda}(T)$. These functions are symmetric polynomials in the transmission eigenvalues $T=\left\{T_{1}, \ldots, T_{n}\right\}$ indexed by partitions $\lambda$. In the group representation theory the Schur functions are the irreducible characters of the unitary group and hence are orthogonal. This orthogonality is quite useful since it means that the coefficients in Schur function expansions are just "Fourier coefficients" and, hence, can be found by integration over the unitary group. It gives an efficient way of calculating the expansion coefficients explicitly, the fact that we exploit in our approach. The Schur functions can be then averaged over the JPDF (2),

$$
\left\langle s_{\lambda}\right\rangle=\int d[T] s_{\lambda}(T) \mathcal{P}_{\beta}(T), \quad d[T] \equiv \prod_{i=1}^{n} d T_{i},
$$

with the help of integration formulas due to Hua. ${ }^{36}$ The Schur function expansions and Hua's integration formulas provide us with the necessary ingredients to compute all moments (or cumulants) of the conductance and/or shot-noise (see Sec. III for the detailed analysis).

In this section, we first give a brief summary of the required facts about partitions and Schur functions, ${ }^{37}$ then develop the systematic way of performing the expansion over Schur functions and finally determine Schur function averages.

\section{A. Partitions and Schur functions}

A partition is a finite sequence $\lambda=\left(\lambda_{1}, \lambda_{2}, \ldots, \lambda_{m}\right)$ of non-negative integers (called parts) in decreasing order $\lambda_{1}$ $\geq \lambda_{2} \geq \ldots \geq \lambda_{m} \geq 0$. The weight of a partition, $|\lambda|$, is the sum of its parts, $|\lambda|=\Sigma_{j} \lambda_{j}$, and the length, $l(\lambda)$, is the number of its nonzero parts. No distinction is made between partitions which differ only by the number of zero parts. Different partitions of weight $m$ represent different ways to write $m$ as the sum of positive integers and can be graphically visualized through the Young diagrams. For example, one has only one partition $\lambda=(1)$ in the trivial case of $m=1$, two partitions $\lambda=(2,0),(1,1)$ for $m=2$, three partitions $\lambda=(3,0,0),(2,1,0),(1,1,1)$ for $m=3$, etc.

For any partition $\lambda$ of length $l(\lambda) \leq n$, one can define a symmetric polynomial $s_{\lambda}$ in $n$ variables $x_{1}, \ldots, x_{n}$ as follows:

$$
s_{\lambda}\left(x_{1}, \ldots, x_{n}\right)=\frac{\operatorname{det}\left\{x_{i}^{\lambda_{j}+n-j}\right\}_{i, j=1}^{n}}{\operatorname{det}\left\{x_{i}^{n-j}\right\}_{i, j=1}^{n}} .
$$

The denominator here is nothing else but the Vandermonde determinant $\Delta(x)=\Pi_{i<j}\left(x_{i}-x_{j}\right)$. It divides the corresponding factor in the nominator, leaving the quotient as a homogeneous polynomial in the $x_{j}$ 's of degree $m=|\lambda|$. These polynomials $s_{\lambda}$ are called the Schur functions. For one-part partitions, $\lambda=(r)$, Schur functions are just the complete symmetric functions, $s_{(r)}(x)=h_{r}$, while for partitions which have no parts other than zero or one, $\lambda=(1, \ldots, 1) \equiv\left(1^{r}\right)$, the Schur functions $s_{\left(1^{r}\right)}$ are the elementary symmetric functions $e_{r}(x)$. This can be verified directly from Eq. (5). It should be noted that the Schur functions corresponding to the partitions of $m$ form a basis in the space of homogeneous symmetric polynomials of degree $m$, so that any homogeneous symmetric polynomial can be written as a linear combination of Schur functions.

The Schur functions of matrix argument that we shall use below are defined by the right-hand side (rhs) in Eq. (5) evaluated at the eigenvalues of the matrix. Taking as an example the $n \times n$ matrix $T=t t^{\dagger}$ of transmission probabilities, one has 


$$
s_{\lambda}(T)=s_{\lambda}\left(T_{1}, \ldots, T_{n}\right)
$$

where $T_{1}, \ldots, T_{n}$ are exactly the transmission eigenvalues that appear in Eq. (2). Although not apparent from this definition, the Schur functions of matrix argument are polynomials in the matrix entries ${ }^{38}$ and, obviously, $s_{\lambda}(T)$ $=s_{\lambda}\left(X T X^{-1}\right)$ for any nondegenerate matrix $X$.

\section{B. Schur function expansions}

In order to determine the moments of the conductance and shot-noise along the lines explained above, one needs to expand the powers of these quantities in Schur functions. To this end, it is more convenient to work with the corresponding generating functions $e^{t \Sigma T_{j}}$ or $e^{t \Sigma T_{j}\left(1-T_{j}\right)}$. These functions belong to the general class of multiplicative symmetric functions, where the coefficients of the Schur function expansion

$$
F(x) \equiv \prod_{j} f\left(x_{j}\right)=\sum_{\lambda} c_{\lambda}^{(f)} s_{\lambda}(x)
$$

can be determined explicitly provided that the function $f$ is analytic in a neighborhood of $|x|=1$ in the complex $x$-plane (see, e.g., Appendix in Ref. 39). Indeed, thinking of the $x_{j}$ 's as of the eigenvalues of a unitary matrix $U$, one can write

$$
F(U)=\sum_{\lambda} c_{\lambda}^{(f)} s_{\lambda}(U)
$$

The main advantage of going unitary is the orthogonality of Schur functions $[d \mu(U)$ is the normalized Haar measure]:

$$
\int_{U(n)} d \mu(U) s_{\lambda}(U) s_{\mu}^{*}(U)=\delta_{\lambda, \mu},
$$

which is a fact from the theory of group representations. One now recognizes a "Fourier series" in Eq. (7) and, hence,

$$
c_{\lambda}^{(f)}=\int_{U(n)} d \mu(U) F(U) s_{\lambda}^{*}(U) .
$$

The integral on the rhs in Eq. (9) is a standard one in RMT. To evaluate it, one first transforms it to the eigenvalues $e^{i \theta_{1}}, \ldots, e^{i \theta_{n}}$ of the unitary matrix $U$. The corresponding Jacobian is $\left|\Delta\left(e^{i \theta}\right)\right|^{2}$, canceling the denominator in the Schur function $s_{\lambda}^{*}\left(e^{i \theta}\right)$. The resulting integral can then be evaluated with the help of the Gram-Andreief identity, ${ }^{40}$ yielding

$$
c_{\lambda}^{(f)}=\operatorname{det}\left\{\int_{0}^{2 \pi} \frac{d \theta}{2 \pi} f\left(e^{i \theta}\right) e^{-i \theta\left(\lambda_{k}-k+l\right)}\right\}_{k, l=1}^{n} .
$$

In view of the analyticity one can abandon the restriction $|x|=1$. Writing $f(x)=\sum_{j} \tau_{j} x^{j}$, one brings the Schur function expansion (6) and (10) to the following general form:

$$
\begin{gathered}
\prod_{i=1}^{n}\left(\sum_{j=-\infty}^{+\infty} \tau_{j} x_{i}^{j}\right)=\sum_{\lambda} c_{\lambda}(\tau) s_{\lambda}(x), \\
c_{\lambda}(\tau) \equiv \operatorname{det}\left\{\tau_{\lambda_{k}-k+l}\right\}_{k, l=1}^{n} .
\end{gathered}
$$

The summation here is over all partitions $\lambda$ of length $n$ or less, including empty partition (0) for which $s_{\lambda}=1$. Expan- sion (10) was also obtained by Balantekin ${ }^{41}$ by algebraic manipulations.

\section{Schur function averages}

Hua in his book ${ }^{36}$ evaluated many useful matrix integrals. The following two are relevant in the context of our work:

$$
\left\langle s_{\lambda}\right\rangle_{\beta=2}=\prod_{j=1}^{n} \frac{\Gamma(j+1) \Gamma\left(\lambda_{j}+n-j+\alpha\right)}{\Gamma\left(\lambda_{j}+2 n-j+\alpha\right)} \prod_{1 \leq i<j \leq n}\left(\lambda_{i}-\lambda_{j}-i+j\right)
$$

and

$$
\left\langle s_{\lambda}\right\rangle_{\beta=1}=\frac{2^{n} n ! \Pi_{1 \leq i<j \leq n}\left(\lambda_{i}-\lambda_{j}-i+j\right)}{\prod_{1 \leq i \leq j \leq n}\left(\lambda_{i}+\lambda_{j}+2 n+2 \alpha-i-j\right)},
$$

where the average $\left\langle s_{\lambda}\right\rangle_{\beta}$ is over the JPDF (2), as in Eq. (4). If $\lambda=(0)$ then $s_{\lambda}=1$ and both integrals follow from the Selberg integral. ${ }^{5}$ It should be noted that the rhs in Eq. (12) is exactly Selberg's expression and the rhs in Eq. (13) can be manipulated to the one obtained by Selberg with the help of the duplication formula for the Gamma function. For nonempty partitions $\lambda$, the integral in Eq. (12) is a particular case of the Kadell-Kaneko-Yan generalization ${ }^{42-44}$ of the Selberg integral. However, the integral in Eq. (13) is different, as the Kadell-Kaneko-Yan generalization of the Selberg integral for $\beta=1$ involves zonal polynomials.

Hua's identities are valid for arbitrary continuous $\alpha>0$. Specifying further to our case of $\alpha=\frac{\beta}{2}\left(\left|N_{1}-N_{2}\right|+1\right)$ and $N$ $\equiv N_{1}+N_{2}$, we arrive after a simple algebra at

$$
\left\langle s_{\lambda}\right\rangle_{\beta=2}=c_{\lambda} \prod_{j=1}^{l(\lambda)} \frac{\left(\lambda_{j}+N_{1}-j\right) !}{\left(N_{1}-j\right) !} \frac{\left(\lambda_{j}+N_{2}-j\right) !}{\left(N_{2}-j\right) !} \frac{(N-j) !}{\left(\lambda_{j}+N-j\right) !},
$$

where we have introduced the coefficient ${ }^{45}$

$$
c_{\lambda}=\frac{\prod_{1 \leq i<j \leq l(\lambda)}\left(\lambda_{i}-i-\lambda_{j}+j\right)}{\prod_{j=1}^{l(\lambda)}\left[l(\lambda)+\lambda_{j}-j\right] !},
$$

and

$$
\begin{aligned}
\left\langle s_{\lambda}\right\rangle_{\beta=1}= & c_{\lambda} \prod_{j=1}^{l(\lambda)} \frac{\left(\lambda_{j}+N_{2}-j\right) !}{\left(N_{2}-j\right) !} \\
& \times \prod_{1 \leq i \leq j \leq N_{2}}\left(\frac{N+1-i-j}{N+1+\lambda_{i}+\lambda_{j}-i-j}\right) .
\end{aligned}
$$

The symmetry between $N_{1}$ and $N_{2}$ is not apparent in Eq. (16). One can rearrange the terms in the second product on 
the rhs in Eq. (16) to make this symmetry apparent

$$
\begin{aligned}
\left\langle s_{\lambda}\right\rangle_{\beta=1}= & c_{\lambda} \prod_{j=1}^{l(\lambda)} \frac{\left(\lambda_{j}+N_{1}-j\right) !}{\left(N_{1}-j\right) !} \frac{\left(\lambda_{j}+N_{2}-j\right) !}{\left(N_{2}-j\right) !} \\
& \times \prod_{1 \leq i \leq j \leq l(\lambda)} \frac{N+1-i-j}{N+1+\lambda_{i}+\lambda_{j}-i-j} \\
& \times \prod_{i=1}^{l(\lambda)} \frac{[N-l(\lambda)-i] !}{\left[\lambda_{i}+N-l(\lambda)-i\right] !}
\end{aligned}
$$

We note that the obtained expressions for $\left\langle s_{\lambda}\right\rangle$ in terms of $N_{1}$ and $N_{2}$ yield zero if the length of the partition $\lambda$ is greater than $n=\min \left(N_{1}, N_{2}\right)$ so when averaging Schur function expansions one need not bother about the restriction $l(\lambda) \leq n$.

The Schur function average for $\beta=2$ in terms of the channel numbers $N_{1}$ and $N_{2}$, Eq. (14), has a simple structure, being a ratio of polynomials

$$
\left\langle s_{\lambda}\right\rangle_{\beta=2}=c_{\lambda} \prod_{j=1}^{|\lambda|} \frac{\left(N_{1}-a_{j}\right)\left(N_{2}-a_{j}\right)}{\left(N-a_{j}\right)}
$$

where the $a_{j}$ 's are integers. Expression (17) for $\left\langle s_{\lambda}\right\rangle_{\beta=1}$ is less revealing. We found it useful to have the Schur function averages tabulated, see Table I for averages corresponding to partitions of $m, m=1, \ldots, 5$. This table suggests ${ }^{46}$ that

$$
\left\langle s_{\lambda}\right\rangle_{\beta=1}=c_{\lambda} \prod_{j=1}^{|\lambda|} \frac{\left(N_{1}-a_{j}\right)\left(N_{2}-a_{j}\right)}{\left(N-b_{j}\right)},
$$

where the $a_{j}$ are the same as in Eq. (18a) and $b_{j}$ 's are also integers. It would be generally desirable to understand the nature of the cancellations in Eq. (17) and to find a rule relating $b_{j}$ to $\lambda$.

\section{MOMENTS AND CUMULANTS OF THE CONDUCTANCE AND SHOT-NOISE}

We now apply the results obtained in the previous two sections to calculate the moments of the conductance and shot-noise in a closed form. The final expressions involve summation over all partitions of $r$ in the case of conductance and $2 r$ in the case of shot-noise, with $r$ being the order of the moment. The cumulants $\kappa_{r}$ can be obtained from the moments $\mu_{r}$ with the help of the well-known recursion

$$
\kappa_{r}=\mu_{r}-\sum_{j=1}^{r-1}\left(\begin{array}{c}
r-1 \\
r-j
\end{array}\right) \mu_{r-j} \kappa_{j} .
$$

This method is well suited for analytic computations of lower order cumulants and also can be straightforwardly implemented in a computer algebra system for computations of higher-order cumulants symbolically. Since a number of the partitions of the given $r$ grows $\sim \exp \{\pi \sqrt{2 r / 3}\} /(4 r \sqrt{3})$ only asymptotically at $r \rightarrow \infty$ (i.e., slower than pure exponential), our method is very efficient for computing the cumulants up to reasonably large orders, as discussed below.

\section{A. Conductance}

The moments of the conductance can be obtained from the generating function

$$
F_{g}(t)=\left\langle e^{t \Sigma_{i} T_{i}}\right\rangle
$$

The desired Schur function expansion for the exponential function $e^{\Sigma_{i} T_{i}}$ can be read from Eq. (11) by choosing $\tau_{j}$ $=1 / j$ ! there. Throughout this paper we use the convention that $1 / j !=0$ for $j<0$. The factorial determinant in Eq. (11) can be evaluated by elementary transformations on its rows or columns and the answer turns out to be exactly the coefficient $c_{\lambda}$ introduced in Eq. (15). Recalling that the Schur functions are homogeneous, thus $s_{\lambda}(t T)=t^{\lambda \mid} s_{\lambda}(T)$, one turns the Schur function expansion of the moment generating function into the following series in powers of $t$ :

$$
F_{g}(t)=\sum_{r=0}^{\infty} t^{r} \sum_{|\lambda|=r} c_{\lambda}\left\langle s_{\lambda}\right\rangle .
$$

The second sum on the right is over all partitions of $r,|\lambda|$ $=\Sigma_{j} \lambda_{j}=r$. From Eq. (21) one easily obtains all moments of the conductance:

$$
\left\langle g^{r}\right\rangle=r ! \sum_{|\lambda|=r} c_{\lambda}\left\langle s_{\lambda}\right\rangle, \quad r=1,2, \ldots, .
$$

For $\beta=2$ this expression together with Eqs. (14) and (15) reproduces the recent result of Novaes. ${ }^{25}$ It is also worth noting here that the corresponding exact results for the charge cumulants (related to the moments $\left\langle T_{1}^{r}\right\rangle$ ), which were obtained in this paper at $\beta=2$, can be straightforwardly generalized to the case of $\beta=1$ by making use of Eq. (17) found above.

With Eq. (22) in hand, one can obtain cumulants by applying recursion (19). On this way we have successfully reproduced the first four cumulants which have been obtained before (exactly for any $\beta$ ). For the reference purpose, we state explicitly the conductance variance ${ }^{13}$

$$
\frac{\operatorname{var}(g)}{\langle g\rangle}=\left\{\begin{array}{ll}
\frac{2\left(N_{1}+1\right)\left(N_{2}+1\right)}{N(N+1)(N+3)}, & \beta=1 \\
\frac{N_{1} N_{2}}{(N-1) N(N+1)}, & \beta=2
\end{array},\right.
$$

with $\langle g\rangle=N_{1} N_{2} /\left(N+\frac{2}{\beta}-1\right)$ being the conductance average, and the third cumulant ${ }^{12}$

$$
\frac{\left\langle\left\langle g^{3}\right\rangle\right\rangle}{\operatorname{var}(g)}=\left\{\begin{array}{cl}
\frac{4\left[1-\left(N_{1}-N_{2}\right)^{2}\right]}{(N-1)(N+1)(N+5)}, & \beta=1 \\
-\frac{2\left(N_{1}-N_{2}\right)^{2}}{(N-2) N(N+2)}, & \beta=2
\end{array},\right.
$$

which is a measure of the skewness of the probability distribution. An explicit expression for the fourth cumulant is quite lengthy for arbitrary $N_{1,2}$ and the corresponding large $N$ expansion can be found in Ref. 12 (see also below). However, in the particular case of $N_{1}=N_{2}=n$, it can be simplified further to the following compact form: 
TABLE I. Schur function expansion $\left\langle\left(\Sigma T_{j}\right)^{m}\right\rangle_{\beta}=m ! \Sigma c_{\lambda}\left\langle s_{\lambda}\right\rangle_{\beta}$ for $\beta=1,2$.

\begin{tabular}{|c|c|c|c|c|}
\hline$m$ & Partition $\lambda$ & $\left\langle s_{\lambda}\right\rangle_{\beta=2}$ & $\frac{\left\langle s_{\lambda}\right\rangle_{\beta=1}}{\left\langle s_{\lambda}\right\rangle_{\beta=2}}$ & $c_{\lambda}$ \\
\hline \multirow{2}{*}{1} & \multirow{2}{*}{ (1) } & $N_{1} N_{2}$ & $N$ & \multirow{2}{*}{1} \\
\hline & & $N$ & $N+1$ & \\
\hline \multirow[t]{4}{*}{2} & \multirow{2}{*}{$(2,0)$} & $\underline{N_{1}\left(N_{1}+1\right) N_{2}\left(N_{2}+1\right)}$ & $\underline{N+1}$ & $\underline{1}$ \\
\hline & & $2 N(N+1)$ & $N+3$ & 2 \\
\hline & \multirow{2}{*}{$(1,1)$} & $\underline{N_{1}\left(N_{1}-1\right) N_{2}\left(N_{2}-1\right)}$ & $\underline{N-1}$ & $\underline{1}$ \\
\hline & & $2 N(N-1)$ & $\overline{N+1}$ & $\overline{2}$ \\
\hline \multirow[t]{6}{*}{3} & \multirow{2}{*}{$(3,0,0)$} & $N_{1}\left(N_{1}+1\right)\left(N_{1}+2\right) N_{2}\left(N_{2}+1\right)\left(N_{2}+2\right)$ & $N+2$ & $\underline{1}$ \\
\hline & & $6 N(N+1)(N+2)$ & $\overline{N+5}$ & $\overline{6}$ \\
\hline & \multirow{2}{*}{$(2,1,0)$} & $\underline{\left(N_{1}-1\right) N_{1}\left(N_{1}+1\right)\left(N_{2}-1\right) N_{2}\left(N_{2}+1\right)}$ & $N$ & 1 \\
\hline & & $3(N-1) N(N+1)$ & $N+3$ & 3 \\
\hline & \multirow{2}{*}{$(1,1,1)$} & $\underline{\left(N_{1}-2\right)\left(N_{1}-1\right) N_{1}\left(N_{2}-2\right)\left(N_{2}-1\right) N_{2}}$ & $N-2$ & 1 \\
\hline & & $6(N-2)(N-1) N$ & $\overline{N+1}$ & $\overline{6}$ \\
\hline \multirow[t]{10}{*}{4} & \multirow{2}{*}{$(4,0,0,0)$} & $N_{1}\left(N_{1}+1\right)\left(N_{1}+2\right)\left(N_{1}+3\right) N_{2}\left(N_{2}+1\right)\left(N_{2}+2\right)\left(N_{2}+3\right)$ & $N+3$ & 1 \\
\hline & & $24 N(N+1)(N+2)(N+3)$ & $\overline{N+7}$ & $\overline{24}$ \\
\hline & \multirow{2}{*}{$(3,1,0,0)$} & $\left(N_{1}-1\right) N_{1}\left(N_{1}+1\right)\left(N_{1}+2\right)\left(N_{2}-1\right) N_{2}\left(N_{2}+1\right)\left(N_{2}+2\right)$ & $\underline{N+1}$ & $\underline{1}$ \\
\hline & & $8(N-1) N(N+1)(N+2)$ & $N+5$ & 8 \\
\hline & \multirow{2}{*}{$(2,2,0,0)$} & $\left(N_{1}-1\right) N_{1}^{2}\left(N_{1}+1\right)\left(N_{2}-1\right) N_{2}^{2}\left(N_{2}+1\right)$ & $(N-1) N^{2}$ & 1 \\
\hline & & $12(N-1) N^{2}(N+1)$ & $(N-2)(N+2)(N+3)$ & $\overline{12}$ \\
\hline & \multirow{2}{*}{$(2,1,1,0)$} & $\left(N_{1}-2\right)\left(N_{1}-1\right) N_{1}\left(N_{1}+1\right)\left(N_{2}-2\right)\left(N_{2}-1\right) N_{2}\left(N_{2}+1\right)$ & $\underline{N-1}$ & 1 \\
\hline & & $8(N-2)(N-1) N(N+1)$ & $N+3$ & 8 \\
\hline & \multirow{2}{*}{$(1,1,1,1)$} & $\left(N_{1}-3\right)\left(N_{1}-2\right)\left(N_{1}-1\right) N_{1}\left(N_{2}-3\right)\left(N_{2}-2\right)\left(N_{2}-1\right) N_{2}$ & $\underline{N-3}$ & 1 \\
\hline & & $24(N-3)(N-2)(N-1) N$ & $N+1$ & 24 \\
\hline \multirow[t]{14}{*}{5} & \multirow{2}{*}{$(5,0,0,0,0)$} & $N_{1}\left(N_{1}+1\right)\left(N_{1}+2\right)\left(N_{1}+3\right)\left(N_{1}+4\right) N_{2}\left(N_{2}+1\right)\left(N_{2}+2\right)\left(N_{2}+3\right)\left(N_{2}+4\right)$ & $\underline{N+4}$ & 1 \\
\hline & & $120 N(N+1)(N+2)(N+3)(N+4)$ & $\overline{N+9}$ & 120 \\
\hline & \multirow{2}{*}{$(4,1,0,0,0)$} & $\underline{\left(N_{1}-1\right) N_{1}\left(N_{1}+1\right)\left(N_{1}+2\right)\left(N_{1}+3\right)\left(N_{2}-1\right) N_{2}\left(N_{2}+1\right)\left(N_{2}+2\right)\left(N_{2}+3\right)}$ & $\underline{N+2}$ & 1 \\
\hline & & $30(N-1) N(N+1)(N+2)(N+3)$ & $\overline{N+7}$ & $\overline{30}$ \\
\hline & \multirow{2}{*}{$(3,2,0,0,0)$} & $\left(N_{1}-1\right) N_{1}^{2}\left(N_{1}+1\right)\left(N_{1}+2\right)\left(N_{2}-1\right) N_{2}^{2}\left(N_{2}+1\right)\left(N_{2}+2\right)$ & $(N-1) N(N+2)$ & 1 \\
\hline & & $24(N-1) N^{2}(N+1)(N+2)$ & $\overline{(N-2)(N+3)(N+5)}$ & $\overline{24}$ \\
\hline & \multirow{2}{*}{$(3,1,1,0,0)$} & $\underline{\left(N_{1}-2\right)\left(N_{1}-1\right) N_{1}\left(N_{1}+1\right)\left(N_{1}+2\right)\left(N_{2}-2\right)\left(N_{2}-1\right) N_{2}\left(N_{2}+1\right)\left(N_{2}+2\right)}$ & $N$ & 1 \\
\hline & & $20(N-2)(N-1) N(N+1)(N+2)$ & $\overline{N+5}$ & $\overline{20}$ \\
\hline & \multirow{2}{*}{$(2,2,1,0,0)$} & $\left(N_{1}-2\right)\left(N_{1}-1\right) N_{1}^{2}\left(N_{1}+1\right)\left(N_{2}-2\right)\left(N_{2}-1\right) N_{2}^{2}\left(N_{2}+1\right)$ & $(N-2)(N-1) N$ & 1 \\
\hline & & $24(N-2)(N-1) N^{2}(N+1)$ & $\overline{(N-3)(N+2)(N+3)}$ & $\overline{24}$ \\
\hline & \multirow{2}{*}{$(2,1,1,1,0)$} & $\left(N_{1}-3\right)\left(N_{1}-2\right)\left(N_{1}-1\right) N_{1}\left(N_{1}+1\right)\left(N_{2}-3\right)\left(N_{2}-2\right)\left(N_{2}-1\right) N_{2}\left(N_{2}+1\right)$ & $N-2$ & 1 \\
\hline & & $30(N-3)(N-2)(N-1) N(N+1)$ & $N+3$ & 30 \\
\hline & \multirow{2}{*}{$(1,1,1,1,1)$} & $\left(N_{1}-4\right)\left(N_{1}-3\right)\left(N_{1}-2\right)\left(N_{1}-1\right) N_{1}\left(N_{2}-4\right)\left(N_{2}-3\right)\left(N_{2}-2\right)\left(N_{2}-1\right) N_{2}$ & $N-4$ & 1 \\
\hline & & $120(N-4)(N-3)(N-2)(N-1) N$ & $N+1$ & 120 \\
\hline
\end{tabular}

$$
\frac{\left\langle\left\langle g^{4}\right\rangle\right\rangle}{\operatorname{var}(g)}=\left\{\begin{array}{cl}
-\frac{3\left(4 n^{4}+20 n^{3}+43 n^{2}+53 n+24\right)}{(n+1)(2 n-1)(2 n+1)^{2}(2 n+3)(2 n+5)(2 n+7)}, & \beta=1 \\
\frac{3}{2(2 n-3)(2 n-1)(2 n+1)(2 n+3)}, & \beta=2
\end{array} .\right.
$$




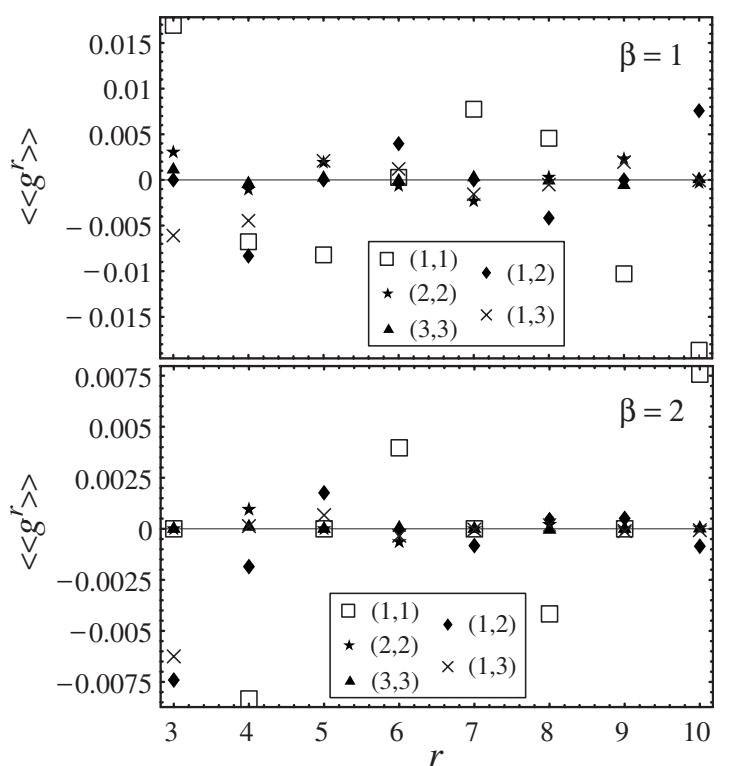

FIG. 1. The third to tenth cumulants of the conductance for chaotic cavities with a few open channels in the case of preserved $(\beta=1)$ or broken $(\beta=2)$ time-reversal symmetry. The corresponding values $\left(N_{1}, N_{2}\right)$ of the channel numbers are as indicated in the legend.

Let us now discuss higher cumulants of the conductance, $\left\langle\left\langle g^{r}\right\rangle\right\rangle$. Their explicit expressions are cumbersome and we consider mainly the physically interesting cases of the small or large channel numbers. In the quantum regime of a few open channels, we have found that these cumulants do not show a pronounced decay with increasing $r$ (see Fig. 1). In this case, the distribution function is strongly non-Gaussian. However, as the number of channels in the both leads increases, the system approaches the semiclassical ("metallic") regime where one should expect ${ }^{47}$ the following dependence of the cumulants on the total number of channels, $N=N_{1}$ $+N_{2}$ :

$$
\left\langle\left\langle g^{r}\right\rangle\right\rangle \sim\langle g\rangle^{2-r} \sim N^{2-r} .
$$

The same scaling is generally applicable to any linear statistic on transmission eigenvalues (e.g., shot-noise), implying a Gaussian distribution in the limit $N \rightarrow \infty$ (Refs. 48 and 49) (see, however, the next section for discussion).

We have performed the asymptotic analysis of our exact RMT expressions in the limit when both $N_{1,2} \gg 1$. To this end, we have employed symbolic computations of conductance cumulants in Mathematica and found that the leadingorder term in the $1 / N$ expansion of the $r$-th cumulant, $r \geq 3$, has the following general structure:

$$
\frac{\left\langle\left\langle g^{r}\right\rangle\right\rangle}{\langle g\rangle} \simeq \frac{(r-1) !}{(\beta / 2)^{r-1}} \frac{N_{1} N_{2}\left(N_{1}-N_{2}\right)^{2}}{N^{3(r-1)}} G_{2(r-3)},
$$

where $G_{m}\left(N_{1}, N_{2}\right)$ is an independent of $\beta$ homogeneous symmetric polynomial of order $m$, (see Table II for the first four ones). Equation (27) has been verified for the first eight cumulants and we are putting forward the conjecture that it holds generally for all $r \geq 3$. The expression on the rhs in Eq. (27) is of the order of $N^{1-r}$, being in agreement with the above estimate (26) obtained within a different approach
TABLE II. The first four polynomial $G_{m}\left(N_{1}, N_{2}\right)$, Eq. (27).

\begin{tabular}{cc}
\hline \hline$r$ & $G_{2(r-3)}\left(N_{1}, N_{2}\right)$ \\
\hline 3 & 1 \\
4 & $N_{1}^{2}-4 N_{1} N_{2}+N_{2}^{2}$ \\
5 & $N_{1}^{4}-10 N_{1}^{3} N_{2}+22 N_{1}^{2} N_{2}^{2}-10 N_{1} N_{2}^{3}+N_{2}^{4}$ \\
6 & $N_{1}^{6}-18 N_{1}^{5} N_{2}+88 N_{1}^{4} N_{2}^{2}-150 N_{1}^{3} N_{2}^{3}+\left(N_{1} \rightleftarrows N_{2}\right)$ \\
\hline
\end{tabular}

(weak localization diagrammatics). The next-to-leadingorder term in the $1 / N$ expansion of $\left\langle\left\langle g^{r}\right\rangle\right\rangle /\langle g\rangle$, the so-called weak localization correction, is of the order of $N^{-r}$. It vanishes for systems with broken time-reversal symmetry $(\beta$ $=2$ ). Further terms in this $1 / N$ expansion can be easily computed as well if necessary.

In the special case of symmetric cavities, $N_{1}=N_{2}=n$, the leading term (27) in the $1 / N$ expansion of the cumulants vanishes for all $r \geq 3$ and so does the next-to-leading term of the expansion of any odd cummulant (independently of $\beta$, it contains a factor $\left(N_{1}-N_{2}\right)^{2}$ explicitly). This indicates that the Gaussian distribution is approached in this case much faster as compared to Eqs. (26) and (27).

Generally, we note that in the symmetric case all odd cumulants at $\beta=2$ must vanish identically, as it follows by the simple symmetry argument ${ }^{12}$ [indeed, the joint distribution (2) becomes then symmetric under the change of all $T_{j}$ $\rightarrow 1-T_{j}$ implying the symmetry of the conductance distribution about its mean $\left.\frac{n}{2}\right]$. It has been recently checked ${ }^{25}$ that representation (22) at $\beta=2$ satisfies this property. For the even cumulants, the $1 / n$ expansion of our exact expressions gives the following leading term at $n \gg 1(k \geq 2)$ :

$$
\left\langle\left\langle g^{2 k}\right\rangle\right\rangle_{\beta=2} \simeq \frac{(2 k-1) !}{4(4 n)^{2 k}}
$$

that agrees with the recent result by Osipov and Kanzieper ${ }^{26}$ obtained by a completely different method. In the case of $\beta=1$, we have found with the help of symbolic computations in Mathematica that

$$
\left\langle\left\langle g^{r}\right\rangle\right\rangle_{\beta=1} \simeq \frac{(r-1) !}{4(2 n)^{r}} \times\left\{\begin{array}{cc}
1, & \text { odd } r \\
\frac{-2 n(r-3) ! !}{r ! !}, & \text { even } r
\end{array} .\right.
$$

for $r=3,4, \ldots, 16$. Correspondingly, we put forward the conjecture that Eq. (29) holds for all $r \geq 3$.

\section{B. Shot-noise}

Having an aim to find also the joint moments of the conductance and shot-noise, we consider the generating function for the moments of $a g+p$

$$
F(t, a) \equiv\left\langle e^{t(a g+p)}\right\rangle=\left\langle\prod_{i=1}^{n} e^{t(a+1) T_{i}-t T_{i}^{2}}\right\rangle .
$$

The moment generating function of shot-noise is then simply given by $F_{p}(t)=F(t, 0)$ whereas that of the conductance follows as $F_{g}(t)=\lim _{a \rightarrow \infty} F(t / a, a)$. At finite $a$, the quantity $a g$ 
$+p$ has a physical meaning of the total noise including both thermal and shot-noise contributions, with $a$ being then the known function of the temperature and applied voltage. ${ }^{2}$

The exponential function in Eq. (30) can be expanded in Schur functions $s_{\lambda}(T)$ with the help of the general identity (10). On multiplying two exponential series, one obtains

$$
\begin{gathered}
F(t, a)=\sum_{\lambda} c_{\lambda}(t, a)\left\langle s_{\lambda}\right\rangle, \\
c_{\lambda}(t, a)=\operatorname{det}\left\{\pi_{\lambda_{i}-i+j}(t, a)\right\}_{i, j=1}^{n},
\end{gathered}
$$

where $\pi_{r}(t, a)$ are polynomials in $t$ and $(a+1)$,

$$
\pi_{r}(t, a)=\sum_{k=0}^{\lfloor r / 2\rfloor} \frac{(-1)^{k}(a+1)^{r-2 k} t^{r-k}}{k !(r-2 k) !} .
$$

In order to extract from this the moments of $a g+p$ one needs to expand the coefficients $c_{\lambda}(t, a)$ in powers of $t$. After some algebra (see Appendix A for details) one arrives at the desired expansion

$$
F(t, a)=\sum_{r=0}^{\infty} t^{r} \sum_{m=0}^{r}(-1)^{m}(1+a)^{r-m} \sum_{|\lambda|=r+m} f_{\lambda, m}\left\langle s_{\lambda}\right\rangle,
$$

where

$$
f_{\lambda, m}=\sum_{k_{1}+\ldots+k_{l(\lambda)}=m} \operatorname{det}\left\{\frac{1}{k_{i} !\left(\lambda_{i}-i+j-2 k_{i}\right) !}\right\} .
$$

The determinant on the rhs (34) can be evaluated in terms of the partition $\lambda$ leading to an explicit expression for the coefficients $f_{\lambda, m}$ [see Eq. (A2)]. In the particular case of $m=0$, $f_{\lambda, 0}$ is just the coefficient $c_{\lambda}$ given by Eq. (15). We note that $f_{\lambda, m}$ depend only on $\lambda$ and $m$ and not on $n$. The summation indices $k_{j}$ in Eq. (34) run over all integers from 0 to $m$ and are not subject to any ordering. From expansion (33), one easily finds that the $r$-th moment of the total noise reads as follows:

$$
\left\langle(a g+p)^{r}\right\rangle=r ! \sum_{m=0}^{r}(-1)^{m}(1+a)^{r-m} \sum_{|\lambda|=r+m} f_{\lambda, m}\left\langle s_{\lambda}\right\rangle,
$$

where the second sum is over all partitions of $r+m$. The joint moment $\left\langle g^{k} p^{r-k}\right\rangle$ of the conductance and shot-noise is then given by Eq. (35), with $(1+a)^{r-m}$ being replaced by the binomial coefficient $\left(\begin{array}{c}r-m \\ k\end{array}\right)$. Equation (35) is generally valid at any $\beta$ and complements the relevant recent results for the joint cumulants of the conductance and shot-noise at $\beta=2$ first obtained within a different approach in Ref. 27 It is interesting to note that by setting $a=-1$, one also obtains the moments of the sum of squares of the transmission coefficients:

$$
\left\langle\left(\sum_{i} T_{i}^{2}\right)^{r}\right\rangle=r ! \sum_{|\lambda|=2 r} f_{\lambda, r}\left\langle s_{\lambda}\right\rangle .
$$

To the best of our knowledge the above formulas have not been reported in the literature before.

We now focus on the analysis of the shot-noise cumulants. Expansion (35) successfully reproduces the general $\beta$ results for the shot-noise average ${ }^{9}$

$$
\langle p\rangle=N_{1} N_{2} \frac{\beta}{2} \frac{\operatorname{var}(g)}{\langle g\rangle}
$$

and for the shot-noise variance. ${ }^{12}$ The explicit expression for the later is rather lengthy (see Ref. 12 for the corresponding large $N$ expansion) but turns out to be quite compact in the particular case of $N_{1}=N_{2}=n$ :

$$
\frac{\operatorname{var}(p)}{\langle p\rangle}=\left\{\begin{array}{cc}
\frac{8 n^{5}+60 n^{4}+142 n^{3}+91 n^{2}-49 n-36}{2(n+1)(2 n-1)(2 n+1)(2 n+3)(2 n+5)(2 n+7)}, & \beta=1, \\
\frac{4 n^{4}-9 n^{2}+3}{4 n(2 n-3)(2 n-1)(2 n+1)(2 n+3)}, & \beta=2
\end{array} .\right.
$$

Higher cumulants of shot-noise, $\left\langle\left\langle p^{r}\right\rangle\right\rangle$, similarly to those of the conductance, are nonvanishing when the number of channels is small, implying a strongly non-Gaussian distribution also in this case. In the opposite limit of the large number of channels, $N_{1,2} \gg 1$, we have found the leading term of the $1 / N$ expansion to have the following structure $(r \geq 3)$ :

$$
\frac{\left\langle\left\langle p^{r}\right\rangle\right\rangle}{\langle p\rangle}=\frac{(r-1) !}{(\beta / 2)^{r-1}} \frac{\left(N_{1}-N_{2}\right)^{2}}{N^{5(r-1)}}\left(N_{1}^{2}-4 N_{1} N_{2}+N_{2}^{2}\right)^{3} P_{4(r-3)},
$$

with $P_{m}\left(N_{1}, N_{2}\right)$ being an independent of $\beta$ homogeneous polynomial of order $m$ (see Table III). This results has been verified in Mathematica for the first six cumulants and is conjectured by us to hold for all $r \geq 3$. The next order term of the expansion has been found to have similar structure to that of the conductance (explicit expressions being, of course, different), thus the same conclusions apply for this term, too.

We consider now the particular case of $N_{1}=N_{2}=n$. In contrast to the conductance, both even and odd cumulants of shot-noise are nonvanishing at finite $n$, even for systems with broken time-reversal symmetry $(\beta=2)$. In the limit of $n \gg 1$, the $1 / n$ expansion of our exact expressions suggests the following asymptotic behavior of the $r$-th cumulant of the shotnoise $(r \geq 3)$ :

$$
\left\langle\left\langle p^{r}\right\rangle\right\rangle_{\beta=2} \simeq \frac{(r-1) !}{4(8 n)^{r}}
$$

and 
TABLE III. The first four polynomial $P_{m}\left(N_{1}, N_{2}\right)$, Eq. (38).

\begin{tabular}{cc}
\hline \hline$r$ & $P_{4(r-3)}\left(N_{1}, N_{2}\right)$ \\
\hline 3 & 1 \\
4 & $N_{1}^{4}-16 N_{1}^{3} N_{2}+34 N_{1}^{2} N_{2}^{2}-16 N_{1} N_{2}^{3}+N_{2}^{4}$ \\
5 & $N_{1}^{8}-38 N_{1}^{7} N_{2}+385 N_{1}^{6} N_{2}^{2}-1344 N_{1}^{5} N_{2}^{3}+2008 N_{1}^{4} N_{2}^{4}$ \\
& $+\left(N_{1} \rightleftarrows N_{2}\right)$ \\
6 & $N_{1}^{12}-66 N_{1}^{11} N_{2}+1345 N_{1}^{10} N_{2}^{2}-11680 N_{1}^{9} N_{2}^{3}$ \\
& $+49699 N_{1}^{8} N_{2}^{4}$ \\
& $-114598 N_{1}^{7} N_{2}^{5}+150662 N_{1}^{6} N_{2}^{6}+\left(N_{1} \rightleftarrows N_{2}\right)$ \\
\hline \hline
\end{tabular}

$$
\left\langle\left\langle p^{r}\right\rangle\right\rangle_{\beta=1} \simeq \frac{(r-1) !}{8(4 n)^{r}} \times\left\{\begin{array}{cc}
1, & \text { odd } r \\
\frac{-4 n(r-3) ! !}{r ! !}, & \text { even } r
\end{array} .\right.
$$

Equation (39) agrees with the very recent result ${ }^{27}$ obtained by a different method. We have been able to verify by symbolic computations in Mathematica that Eq. (40) holds up to the 8-th cumulant and, thus, conjecture it to hold for any $r$ $\geq 3$.

\section{DISTRIBUTION FUNCTIONS}

We consider now the distribution function of the conductance, $P_{g}^{(\beta)}(x)=\langle\delta(x-g)\rangle$, and that of shot-noise, $P_{p}^{(\beta)}(x)$ $=\langle\delta(x-p)\rangle$, with $g$ and $p$ being defined in Eq. (1). Explicit expressions for the conductance distribution can be found in the particular cases of $n=1,2$. At $N_{1}=1$ and $N_{2}=K \geq 1$, Eq. (2) readily gives $P_{g, n=1}^{(\beta)}(g)=(\beta K / 2) g^{\beta K / 2-1}$ for $0<g<1$, and zero otherwise. In the case of $N_{1}=2$ and arbitrary $N_{2}=K$ $\geq 2$, the conductance distribution can also be found by performing integrations that feature in the definition [see (B4) in Appendix B], with the final result being

$$
\begin{aligned}
P_{g, n=2}^{(\beta)}(g)= & K^{\beta K-1}\left\{X_{1}-(-1)^{(\beta K-1) / 2} X_{2} \Theta(g-1)\right. \\
& \left.\times \sum_{j=0}^{\beta}\left(\begin{array}{l}
\beta \\
j
\end{array}\right) B_{1-g}\left[\frac{\beta}{2}(K-1)+j, 1-\beta K\right]\right\}
\end{aligned}
$$

for $0<g<2$ and zero otherwise (see Fig. 2). Here $B_{z}(a, b)$ is the incomplete beta function, $\Theta(x)$ stands for the Heaviside step function, and the constants $X_{1,2}$ are given by $X_{1}$ $=\frac{\Gamma[\beta(K+1) / 2+1] \Gamma(\beta K / 2)}{\Gamma(\beta / 2) \Gamma(\beta K)}$ and $X_{2}=\frac{\Gamma[\beta(K+1) / 2+1]}{\Gamma(\beta) \Gamma[\beta(K-1) / 2]}$. Expression $(41)$ holds for arbitrary positive integer $\beta$. In the particular case of $\beta=1$, it can be simplified further, yielding $P_{g, n=2}^{(1)}(g)=\frac{1}{2} K(K$ $+1)\left[\left(\frac{g}{2}\right)^{K-1}-(g-1)^{(K-1) / 2} \Theta(g-1)\right]$, in agreement with Ref. 28.

It is possible to find explicit expressions for the distribution function of the conductance beyond the cases discussed above. However, the final answers become more cumbersome as the channel numbers grow, thus being almost of little practical use. For the shot-noise distribution, the situation is not satisfactory even for small channel numbers: we are not aware of explicit results for the shot-noise distribution except for the simplest case of $N_{1,2}=1 .^{29}$ One practical

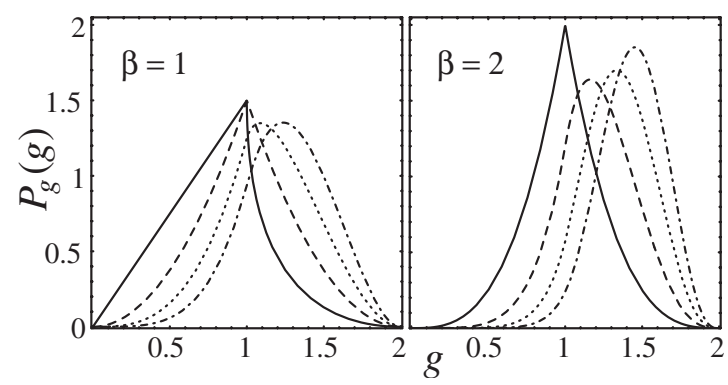

FIG. 2. The conductance distribution (41) for chaotic cavities with preserved $(\beta=1)$ or broken $(\beta=2)$ time-reversal symmetry. The number of channels is fixed to $N_{1}=2$ in one lead and varied in the other, $N_{2}=2,3,4$, or 5 (solid, dashed, dotted or dash-dotted lines, respectively). Nonanalyticity of the distribution (a cusp point at $g=1$ ) becomes less pronounced as $N_{2}$ increases.

way to solve this problem is to construct approximations to the distribution functions in terms of cumulants by making use of the Edgeworth expansion. ${ }^{51}$ It turns out that such approximations are fairly accurate in the bulk of distribution even for small channel numbers.

In the limit $N_{1,2} \gg 1$ the conductance and shot-noise distributions follow the Gaussian law

$$
\phi_{0}(x)=\frac{1}{\sqrt{2 \pi \sigma^{2}}} e^{-(x-\mu)^{2} / 2 \sigma^{2}}
$$

where $\mu$ and $\sigma^{2}$ are the corresponding mean value and variance, respectively. The Edgeworth expansion is a $1 / N$ expansion around the Gaussian law. Denoting the $r$-th cumulant by $\kappa_{r}$, the first correction to the Gaussian law is given by $\phi_{1}(x)=-\frac{1}{3 !} \kappa_{3} \partial^{3} \phi_{0}(x)$ and the next one is given by $\phi_{2}(x)$ $=\left(\frac{1}{4 !} \kappa_{4} \partial^{4}+\frac{10}{6 !} \kappa_{3}^{2} \partial^{6}\right) \phi_{0}(x)$. Higher-order corrections involve higher-order cumulants. ${ }^{52}$ Restricting ourselves to the first four cumulants, we get the following approximation to the distribution functions of interest:

$$
P_{g, p}^{(\beta)}(x) \simeq \sum_{k=0}^{2} \phi_{k}(x) .
$$

The advantage of the Edgworth expansion is that it is a true asymptotic series, with the controlled error [e.g., in our case the error of approximation (43) is estimated to be on the order of $\frac{1}{N^{3}}$. With the higher-order cumulants being readily available from Eqs. (22), (35), and (19), one can easily improve the accuracy of the approximation by adding higherorder corrections if needed.

We found it instructive to compare the above approximation (43) with the exact results which are available for the conductance distribution. To our surprise, we found the Edgeworth approximation to be fairly accurate already for $N_{1}=2$ and $N_{2} \gtrsim 4$ (see Fig. 3). The agreement between the approximate and exact distributions gets even better for $N_{1,2} \geq 3$.

The Edgeworth approximation fails near the edges and also if the distribution has strong singularities. Therefore, it is tempting to look for an alternative and exact representation for the distribution functions of the conductance and shotnoise. We note that at any finite number of channels each of these distributions has a finite support, namely, $0 \leq g \leq n$ 


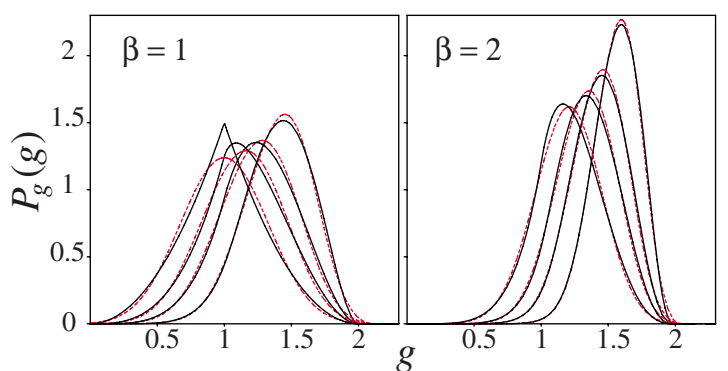

FIG. 3. (Color online) Comparison of the exact conductance distribution (41) (black solid lines) with the Edgeworth approximation (43) (red dashed lines) for chaotic cavities with preserved ( $\beta$ $=1)$ or broken $(\beta=2)$ time-reversal symmetry. The number of channels is fixed to $N_{1}=2$ in one lead and varied in the other, $N_{2}=3,4$, 5 , and 7 (lines from left to right, respectively). The purely Gaussian approximation would produce much stronger systematic deviations, thus being not sufficient at small channel numbers at all.

$\equiv I_{g}$ and $0 \leq p \leq \frac{n}{4} \equiv I_{p}$, being identically zero outside this region. It is, therefore, natural to represent the distribution functions in the following form (henceforth, variable and in$\operatorname{dex} x=g, p)$ :

$$
P_{x}^{(\beta)}(x)=\sum_{m=1}^{\infty} \frac{2}{I_{x}} \sin \left(\frac{m \pi x}{I_{x}}\right) C_{x}^{(\beta)}(m),
$$

as the Fourier series over the interval of support, cf. Schur function expansion (7).

We now show that the Fourier coefficients are given by Pfaffians $^{50}$ as follows:

$$
C_{x}^{(\beta)}(m)=\frac{n !}{\mathcal{N}_{\beta}} \operatorname{Im} \operatorname{Pfaff}\left[A_{x}^{(\beta)}(m)\right] .
$$

Before establishing explicit forms of the antisymmetric matrices $A_{x}^{(\beta)}(m)$, it is useful to note the following. The Fourier coefficients turn out to decay generally as $C_{x}^{(\beta)}(m) \sim m^{-\nu_{x}}$ as $m \rightarrow \infty$, where exponent $\nu_{x}>1$ depends on the case considered. This readily leads to the observation that the everywhere continuous distribution function (44) contains (rather weak) singularities at integer points of division of the support interval $\left[0, I_{x}\right]$, as certain derivatives become discontinuous at these points (see further Ref. 11 for an alternative geometric interpretation of these singularities). Such a nonanalyticity is expected to become less and less pronounced when the number of channels grows, as the bulk of the distribution is described then by a Gaussian law. However, the asymptotic behavior near the edges of support is always characterized by a power law. ${ }^{11}$ Consequently, even at $n \gg 1$ the distribution remains to be weakly singular at the junction of Gaussian and power-law regimes ${ }^{30}$ (see also Ref. 26).

We start the derivation of Eq. (45) with the simplest case of unitary symmetry, $\beta=2$, and consider first the conductance distribution

$$
P_{g}^{(2)}(g)=\frac{1}{\mathcal{N}_{2}} \int d[T] \prod_{j=1}^{n} T_{j}^{\alpha-1} \Delta(T)^{2} \delta\left(g-\sum_{i=1}^{n} T_{i}\right) .
$$

Writing $\Delta(T)=\operatorname{det}\left\{T_{j}^{i-1}\right\}$, with $i, j=1, \ldots, n$, and substituting the Fourier representation of the $\delta$-function, $\delta\left(g-\Sigma_{i} T_{i}\right)$ $=\int \frac{d \omega}{2 \pi} e^{-i \omega g+i \omega \Sigma_{i} T_{i}}$, one can interchange the order of integrations and then apply the Gram-Andreief identity to perform the integrations over $T_{j}$ 's. This readily yields

$$
\begin{gathered}
P_{g}^{(2)}(g)=\frac{n !}{\mathcal{N}_{2}} \int_{-\infty}^{\infty} \frac{d \omega}{2 \pi} e^{-i \omega g} \operatorname{det}\left[\mathcal{A}_{g}^{(2)}(\omega)\right], \\
{\left[\mathcal{A}_{g}^{(2)}(\omega)\right]_{k l}=\int_{0}^{1} d T T^{\alpha+k+l-3} e^{i \omega T} .}
\end{gathered}
$$

Since we know that the conductance distribution has a support only in $[0, n]$ it is actually more convenient to expand $P_{g}^{(2)}(g)$ in functions $\sqrt{2 / n} \sin (m \pi g / n)$, with $m=1,2, \ldots$, which form a complete and orthonormalized set on this interval. Furthermore, due to factorization of the exponential function, one has to express first everything in terms of $\exp \left(i m \pi T_{j} / n\right)$ and then take the imaginary part. As a result, we arrive at the final answer cast in the form of Eq. (45), where

$$
\operatorname{Pfaff}\left[A_{g}^{(2)}(m)\right] \equiv \operatorname{det}\left[\tilde{A}_{g}^{(2)}(m)\right]
$$

and $\widetilde{A}_{g}^{(2)}(m) \equiv \mathcal{A}_{g}^{(2)}\left(\frac{m \pi}{n}\right)$ is the discrete analog of Eq. (46b).

In the case of orthogonal symmetry, $\beta=1$, the derivation goes along the same lines but has to be done separately for even or odd $n$. In the case of even $n$, we first consider the $T_{j}$ 's in a special order $T_{1}<T_{2}<\ldots<T_{n}$ (hence $n$ !). Then it is useful to represent the Vandermonde determinant as a Gaussian integral over two kinds of Grassmann variables and integrate out further one set of them using the method of alternating variables, ${ }^{5}$ see also Ref. 53 for relevant details. The resulting expression acquires then the symplectic (antisymmetric) structure automatically and the remaining average yields Eqs. (44) and (45), with

$$
\begin{aligned}
{\left[A_{g}^{(1)}(m)\right]_{k l}^{\text {even } n}=} & \int_{0}^{1} d T_{1} \int_{0}^{1} d T_{2} \operatorname{sign}\left(T_{2}-T_{1}\right) T_{1}^{\alpha+k-2} \\
& \times T_{2}^{\alpha+l-2} e^{i(m \pi / n)\left(T_{1}+T_{2}\right)} .
\end{aligned}
$$

In the case of odd $n$, one has to increase artificially the number of the Grasmannians by one and proceed as before, with the final result being

$$
\left[A_{g}^{(1)}(m)\right]_{k l}^{\text {odd } n}=\left[\begin{array}{cc}
\tilde{A}_{k l}^{(1)} & B_{k, n+1} \\
-B_{n+1, l} & 0
\end{array}\right] .
$$

Here the $n \times n$ matrix $\widetilde{A}^{(1)}$ is given by Eq. (48), and the $n$-dimensional vector $B$ is

$$
B_{k, n+1}=\int_{0}^{1} d T T^{\alpha+k-2} e^{i(m \pi / n) T}=B_{n+1, k} .
$$

For the sake of completeness, we also state the result in the case of symplectic symmetry, $\beta=4$ :

$$
\left[A_{g}^{(4)}(m)\right]_{k l}=(l-k) \int_{0}^{1} d T T^{\alpha+k+l-4} e^{i(m \pi / n) T},
$$

where now $k, l=1, \ldots, 2 n$. It can be obtained by reducing the fourth power of the Vandermonde determinant to the calcu- 
lation of a Vandermonde determinant with the doubled dimension $2 n$ subject to the additional $\delta$-function constraints for the corresponding pairs of eigenvalues. The rest is as in the orthogonal case above.

We note that it turns out to be possible to find explicit expressions for the conductance distribution in the case of $n=3$ and 4 by evaluating the above Pfaffians analytically and performing the corresponding Fourier transformation exactly.

The expressions derived in this section are also well suited for numerical calculations. It is important to note in this respect that, as the Pfaffian is defined as an analytic square root of the determinant of an antisymmetric matrix $A$, special care has to be taken to decide for the complex determinant which sign has to be chosen. To overcome this difficulty, we outline the following general procedure. First, we multiply the matrix $A$ with the symplectic unit $Z \equiv \operatorname{diag}\{$ $\left.-i \sigma_{2}, \ldots,-i \sigma_{2}\right\}, \sigma_{2}$ being the Pauli matrix. Then we note that the matrix $Z A$ is self-dual, $Z A=A Z$, implying that its eigenvalues come in pairs. Taking the product of all eigenvalues of $Z A$ only once (this is exactly the quaternion determinant of $Z A$ ), we obtain finally the Pfaffian of $A$. As an illustration of this procedure, let us consider the simplest example of calculating the Pfaffian of $\left(\begin{array}{cc}0 & a \\ -a & 0\end{array}\right)=i \sigma_{2} a$ which is equal to $a$. The matrix $\left(-i \sigma_{2}\right)\left(i \sigma_{2} a\right)=\operatorname{diag}\{a, a\}$ has obviously eigenvalues $(a, a)$, so that taking $a$ once yields the Pfaffian $a$. This is exactly the way how Pfaffians can be easily computed numerically.

Generally, one can derive the distribution function of any linear statistic on $T, x=\sum_{j} f_{x}\left(T_{j}\right)$ with a given $f_{x}(T)$, in complete analogy with the above lines. It yields representation (44), where matrices $A_{x}^{(\beta)}(m)$ are given by the above expressions (47)-(51) in which all the factors $\exp \{i(m \pi / n) T\}$ have to be obviously substituted with $\exp \left\{i\left(m \pi / I_{x}\right) f_{x}(T)\right\}$, with $I_{x}$ being the length of the corresponding support interval. In particular, for the case of shot-noise it amounts to substituting there with $\exp \{i(4 m \pi / n) T(1-T)\}$.

\section{ASYMPTOTICS}

The asymptotic behavior of the distribution functions near the edges is characterized by a power-law dependence

$$
P_{x}^{(\beta)}(x) \simeq\left\{\begin{array}{cc}
L_{x} x^{\ell_{x}}, & x \rightarrow 0 \\
R_{x}\left(I_{x}-x\right)^{r_{x}}, & x \rightarrow I_{x}
\end{array},\right.
$$

where both the exponents and the prefactors can be determined exactly at arbitrary $N_{1,2}$ and any $\beta$, as shown below.

In the case of the conductance, the exponents $\ell_{g}$ and $r_{g}$ have been already reported previously, ${ }^{11}$ being given by

$$
\begin{gathered}
\ell_{g}=\alpha n+\frac{\beta}{2}(n-1) n-1 . \\
r_{g}=(n-1)\left(1+\frac{\beta}{2} n\right)
\end{gathered}
$$

To determine the constant $L_{g}$, we consider $P_{g}(g)$ at $g<1$. In this case, the upper limit of the integrations over $T_{i}$ 's in $P_{g}(g)=\left\langle\delta\left(g-\Sigma_{i} T_{i}\right)\right\rangle$ may be replaced with $g$ (due to the $\delta$ function). Scaling further all $T_{i} \rightarrow g T_{i}$ and calculating the
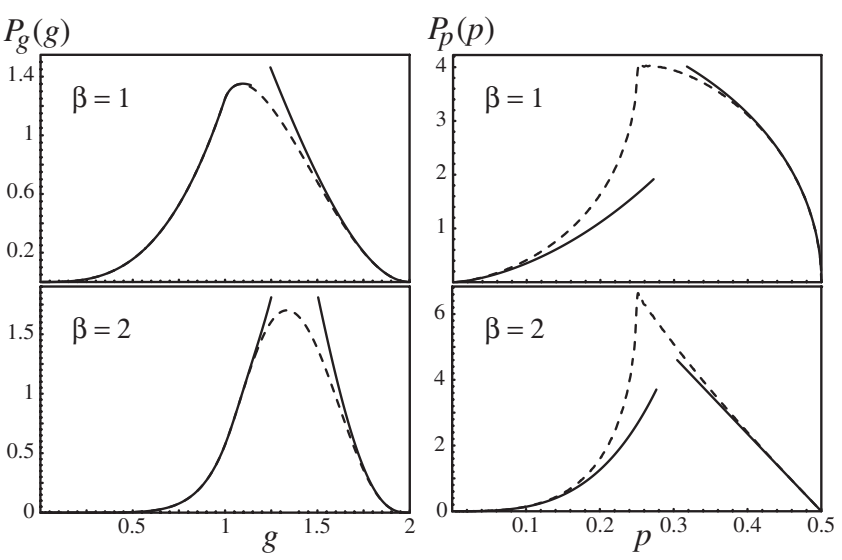

FIG. 4. The distribution of the conductance $\left[P_{g}(g)\right.$, left $]$ and that of shot-noise $\left[P_{p}(p)\right.$, right $]$ for chaotic cavities with preserved $(\beta$ $=1)$ or broken $(\beta=2)$ time-reversal symmetry. The number of channels are $N_{1}=2$ and $N_{2}=4$. Dashed lines correspond to the exact distributions whereas solid lines show the corresponding asymptotic behavior near the edges, see the text for details.

powers of $g$ there, one readily gets $P_{g}(g)=L_{g} g^{\ell_{g}}$, with $L_{g}$ $=\left\langle\delta\left(1-\Sigma T_{i}\right)\right\rangle$. It is worth emphasizing that this is the exact expression of the conductance distribution at $0<g<1$. $^{11,15}$ The integral for $L_{g}$ can be then calculated by the standard RMT methods (see Appendix B) and is given by

$$
L_{g}=\frac{1}{\Gamma\left(\ell_{g}+1\right)} \prod_{j=0}^{n-1} \frac{\Gamma\left[1+\alpha+\frac{\beta}{2}(n+j-1)\right]}{\Gamma\left(1+\frac{\beta}{2} j\right)} .
$$

The behavior of the distribution near the right edge can be analyzed in a similar way, yielding Eq. (52) with

$$
R_{g}=\frac{1}{\Gamma\left(r_{g}+1\right)} \prod_{j=0}^{n-1} \frac{\Gamma\left[1+\alpha+\frac{\beta}{2}(n+j-1)\right]}{\Gamma\left(\alpha+\frac{\beta}{2} j\right)} .
$$

In the case of shot-noise, the corresponding exponents are found to be as follows:

$$
\begin{gathered}
\ell_{p}=\alpha n+\frac{\beta}{4}\left[(n-1)^{2}-\sigma\right]-1, \\
r_{p}=\frac{n}{2}+\frac{\beta}{4}(n-1) n-1,
\end{gathered}
$$

where $\sigma=1$ or 0 for $n$ even or odd, respectively. The above expression for $r_{g}$ was already known ${ }^{11}$ whereas that for $\ell_{p}$ is new, being in agreement with the recent large $n$ result. ${ }^{30}$ The corresponding constants can also be found exactly. We refer to Appendix B for further details, including the discussion of correction factors to the leading asymptotics given above.

As an illustration of the obtained results, Fig. 4 shows a comparison between the exact and asymptotic behavior of the conductance and shot-noise distributions in the case of chaotic cavities with $N_{1}=2$ and $N_{2}=4$ channels.

\section{CONCLUSIONS}

In this work, we have presented a systematic study of statistics of the conductance and shot-noise in chaotic cavities. Our approach is based on expanding symmetric func- 
tions in the transmission eigenvalues, of which the conductance and shot-noise are two examples, in Schur functions and then applying a generalization of Selberg's integral due to Hua to evaluate the averages. This leads to explicit formulas for the conductance and shot-noise cumulants in terms of the channel numbers for chaotic cavities with preserved $(\beta$ $=1)$ or broken $(\beta=2)$ time-reversal symmetry.

For lower order cumulants our formulas reproduce the previously known exact results. We have performed an asymptotic analysis of the cumulants in the regime when the number of channels in both leads increases. It suggests that, generically, the $r$-th cumulant decays as $N^{2-r}$ for $r \geq 3$ with the leading term containing $\left(N_{1}-N_{2}\right)^{2}$ as a factor; see Eqs. (27) and (38). This implies that the convergence to the limiting Gaussian law is faster in the case of symmetric cavities, $N_{1}=N_{2}=n$. In this case, we have been able to analyze the first 16 cumulants of the conductance and the first 8 cumulants of shot-noise in the limit $n \gg 1$, obtaining the leadingorder term in the $1 / n$ expansion explicitly in terms of the cumulant order, $r$, and the channel number, $n$. For the systems with broken time-reversal symmetry, the $r$-th cumulant decays as $1 / n^{r}$ for both conductance and shot-noise, with all the odd cumulants of the conductance being identically zero. Our results in this case, Eqs. (28) and (39), agree with those of Osipov and Kanzieper ${ }^{26,27}$ obtained recently by a completely different method. For systems with preserved timereversal symmetry, we have found that the $r$-th cumulant decays as $1 / n^{r}$ for odd $r$ and $1 / n^{r-1}$ for even $r, r \geq 3$, for both the conductance and shot-noise. This staircase effect in the rate of the cumulant decay seems to be a feature which has not been reported in the literature before. One of its apparent consequences is that the convergence to the limiting Gaussian law is slower for systems with preserved time-reversal symmetry. We have also put forward our explicit formulas (29) and (40) for the higher-order cumulants in the whole range of $r \geq 3$ as a conjecture. Proving this conjecture seems to us an interesting and challenging open problem.

As mentioned above, in the limit when the number of open channels in both leads increases, the conductance and shot-noise distributions are described by the Gaussian law. With higher-order cumulants in hand, one can easily obtain next order corrections to the Gaussian law by making use of the Edgeworth expansion. We have found that such approximations to the distribution function are fairly accurate in the bulk even for small channel numbers. We have also obtained an alternative and exact representation for the distribution functions, in terms of Pfaffians, which is suitable in the whole range of support, including the edges where the distributions have a power-law dependence. Such an asymptotic behavior have been investigated in detail, the powers and corresponding prefactors being determined exactly at any $\beta$ and $N_{1,2}$.

\section{ACKNOWLEDGMENTS}

The authors thank W. Wieczorek for his help with numerical calculations used to produce plots on Fig. 4. The two of us (B.A.K. and D.V.S.) would like to acknowledge the hospitality of the Isaac Newton Institute (Cambridge, U.K.) during their stay there at the program "Mathematics and Physics of Anderson localization: 50 Years After," where this work has been initiated and partly completed. Financial support by the Grant No. SFB/TR12 of DFG (H.-J.S.) and by BRIEF grant (D.V.S.) is acknowledged with thanks.

\section{APPENDIX A: SCHUR FUNCTION EXPANSION, EQ. (30)}

In this Appendix we expand the coefficients $c_{\lambda}(t, a)$ $=\operatorname{det}\left\{\pi_{\lambda_{i}-i+j}(t, a)\right\}$ in powers of $t$. From Eq. (32) and the definition of determinant,

$$
\begin{aligned}
\operatorname{det}\left[\pi_{\lambda_{i}-i+j}(t, a)\right] & =\sum_{\sigma \in S_{n}}(-1)^{|\sigma|} \prod_{i=1}^{n} \sum_{k_{i}} \frac{(-1)^{k_{i}}(1+a)^{\lambda_{i}-i+\sigma(i)-2 k_{i} t^{\lambda_{i}-i+\sigma(i)-k_{i}}}}{k_{i} !\left[\lambda_{i}-i+\sigma(i)-2 k_{i}\right] !} \\
& =\sum_{\sigma \in S_{n}}(-1)^{|\sigma|} \sum_{k_{1}, \ldots, k_{n}}(-1)^{K}(1+a)^{|\lambda|-2 K} t^{|\lambda|-K} \prod_{i=1}^{n} \frac{1}{k_{i} !\left[\lambda_{i}-i+\sigma(i)-2 k_{i}\right] !}
\end{aligned}
$$

where $K=\sum_{i} k_{i}$ and the first sum is over all permutations $\sigma$ in the symmetric group $S_{n}$. Changing the order of summations again, one can fold the sum over permutations into a determinant again. This yields

$$
\begin{aligned}
c_{\lambda}(t, a) & =\sum_{m=0}^{\lfloor|\lambda| / 2\rfloor}(-1)^{m}(1+a)^{|\lambda|-2 m} t^{|\lambda|-m} \\
\times & \sum_{k_{1}+\ldots+k_{n}=m} \operatorname{det}\left\{\frac{1}{k_{i} !\left(\lambda_{i}-i+j-2 k_{i}\right) !}\right\}_{i, j=1}^{n} .
\end{aligned}
$$

The second sum here is exactly the coefficient $f_{\lambda, m}$ that appears in Eq. (30). The determinant in Eq. (A1) can be evalu- ated by elementary transformations on its rows and columns,

$$
\begin{aligned}
& \operatorname{det}\left\{\frac{1}{k_{i} !\left(\lambda_{i}-i+j-2 k_{i}\right) !}\right\}_{i, j=1}^{n} \\
& \quad=\frac{\prod_{1 \leq i<j \leq n}\left(\lambda_{i}-i-\lambda_{j}+j-2 k_{i}+2 k_{j}\right)}{\prod_{j=1}^{n} k_{j} !\left(n+\lambda_{j}-j-2 k_{j}\right) !} .
\end{aligned}
$$

Note that for any $n \geq l(\lambda)$, where $l(\lambda)$ is the length of $\lambda$, one can safely replace $n$ in the above expressions by $l(\lambda)$. Hence, the sum over the $n$ tuples $\left(k_{1}, \ldots, k_{n}\right)$ of integers in Eq. (A1) can be replaced by the sum over the $l(\lambda)$-tuples $\left(k_{1}, \ldots, k_{l(\lambda)}\right)$, yielding 


$$
f_{\lambda, m}=\sum_{k_{1}+\ldots+k_{l(\lambda)}=m} \frac{\Pi_{1 \leq i<j \leq l(\lambda)}\left(\lambda_{i}-i-\lambda_{j}+j-2 k_{i}+2 k_{j}\right)}{\prod_{j=1}^{l(\lambda)} k_{j} !\left[l(\lambda)+\lambda_{j}-j-2 k_{j}\right] !} .
$$

We now substitute the obtained expression for $c_{\lambda}(t, a)$ back in Eq. (31) to obtain

$$
F(t, a)=\sum_{r=0}^{\infty} \sum_{m=0}^{\lfloor r / 2\rfloor}(-1)^{m}(1+a)^{r-2 m} t^{r-m} \sum_{|\lambda|=r} f_{\lambda, m}\left\langle s_{\lambda}\right\rangle_{\beta},
$$

hence the desired Schur function expansion (33) follows after changing the order of summations.

\section{APPENDIX B: ASYMPTOTIC CONSTANTS AND CORRECTION FACTORS}

Conductance, left edge: to calculate $L_{g}=\left\langle\delta\left(1-\Sigma_{j} T_{j}\right)\right\rangle$, we use the following result due to Mehta ${ }^{5}$ (see p. 361):

$$
\begin{aligned}
\int_{0}^{\infty} & \cdots \int_{0}^{\infty} \prod_{j=1}^{n} d x_{j} x_{j}^{\alpha-1}|\Delta(x)|^{\beta} \frac{\Theta\left(1-\sum_{i} x_{i}\right)}{\left(1-\Sigma_{i} x_{i}\right)^{1-\gamma}} \\
= & \frac{\Gamma(\gamma)}{\Gamma\left[\gamma+\alpha n+\frac{\beta}{2} n(n-1)\right]} \prod_{i=1}^{n} \frac{\Gamma\left[\alpha+\frac{\beta}{2}(n-i)\right] \Gamma\left(1+\frac{\beta}{2} i\right)}{\Gamma\left(1+\frac{\beta}{2}\right)} .
\end{aligned}
$$

Making use of the known identity $\lim _{\gamma \rightarrow 0^{+}} \gamma(1-x)^{\gamma-1} \Theta(1$ $-x) \Theta(x)=\delta(1-x)$ and noting that $\gamma \Gamma(\gamma)=\Gamma(1+\gamma) \rightarrow 1$ at $\gamma$ $\rightarrow 0$, one can readily get from Eq. (B1) the following result (valid at any $\beta>0$ ):

$$
\begin{aligned}
\int_{0}^{1} \ldots \int_{0}^{1} d[T] \prod_{j=1}^{n} T_{j}^{\alpha-1}|\Delta(T)|^{\beta} \delta\left(1-\sum_{i} T_{i}\right) \\
\quad=\frac{1}{\Gamma\left[\alpha n+\frac{\beta}{2} n(n-1)\right]} \prod_{j=0}^{n-1} \frac{\Gamma\left(\alpha+\frac{\beta}{2} j\right) \Gamma\left[1+\frac{\beta}{2}(j+1)\right]}{\Gamma\left(1+\frac{\beta}{2}\right)} .
\end{aligned}
$$

After dividing Eq. (B2) with the normalization constant (3), this yields Eq. (54) of the main text.
Conductance, right edge: for considering the limit $g$ $\rightarrow n$ from below, we make a transformation $T_{i} \rightarrow 1-T_{i}$ in the integral for $P_{g}^{(\beta)}(g)=\mathcal{N}_{\beta}^{-1} \int d[T] \Pi_{j}\left(1-T_{j}\right)^{\alpha-1}|\Delta(T)|^{\beta} \delta(n-g$ $-\Sigma_{i} T_{i}$ ) and then consider $T_{i}$ small. Now one sees that in the region $n>g>n-1$ the upper bound of the integral can be replaced by $n-g$. Further rescaling $T_{i} \rightarrow(n-g) T_{i}$ yields

$$
P_{g}^{(\beta)}(g) \simeq R_{g}(n-g)^{(n-1)\left(1+\frac{\beta}{2} n\right)} e^{-(\alpha-1)(n-g)},
$$

where $R_{g}$ is then given by Eq. (55) [use result Eq. (B2) at $\alpha=1$ and divide it by $\left.\mathcal{N}_{\beta}\right]$. The correction factor $e^{-(\alpha-1)(n-g)}$ comes from expanding $\Pi_{i}\left(1-(n-g) T_{i}\right)^{\alpha-1}$ linearly in the exponent. Thus we have systematically expanded the log of the positive quantity $P_{g}^{(\beta)}(g)$ for small $(n-g)$ including the term of order $(n-g)$. Corrections are of relative order $(n-g)^{2}$.

Conductance, $1<g<2$ : since we know $P_{g}^{(\beta)}(g)$ in the interval $0<g<1$ exactly, one can consider asymptotics in the interval $1<g<2$ for $g \rightarrow 1$. To this end, we first arrange the integration variables as $T_{1}<T_{2}<\cdots<T_{n}$, then rescale $T_{k} \rightarrow T_{n} T_{k}$ for $k=1,2, \ldots, n-1$, and finally perform the $T_{n}$ integration. As a result, one arrives at the following exact representation of the conductance distribution at $0<g<2$ :

$$
P_{g}^{(\beta)}(g)=g^{\alpha n+\frac{\beta}{2}(n-1) n-1}\left[L_{g}-\widetilde{P}(g)\right],
$$

where $\quad \widetilde{P}(g)=\left(n / \mathcal{N}_{\beta}\right) \int d[T]|\Delta(T)|^{\beta} \prod_{i=1}^{n-1} T_{i}^{\alpha-1}\left(1-T_{i}\right)^{\beta}(1$ $\left.+\sum_{i=1}^{n-1} T_{i}\right)^{-n \alpha-n(n-1) \beta / 2} \Theta\left(g-1-\sum_{i=1}^{n-1} T_{i}\right)$. In the particular case of $n=2$, this integral is one-dimensional and can be easily evaluated explicitly, resulting in Eq. (41). At any $n>2, \tilde{P}(g)$ can be handled with the same methods as before, yielding the following asymptotic behavior at small $g-1$ :

$$
\widetilde{P}(g) \simeq \Theta(g-1) H(g-1)^{\alpha(n-1)+\frac{\beta}{2}(n-2)(n-1)} e^{-J(g-1)},
$$

where the constants $H$ and $J$ are given by

$$
H=\frac{n\left[\alpha+\frac{\beta}{2}(n-1)\right]}{\Gamma\left[1+\alpha(n-1)+\frac{\beta}{2}(n-2)(n-1)\right]} \prod_{j=1}^{n-1} \frac{\Gamma\left[1+\alpha+\frac{\beta}{2}(n+j-1)\right]}{\Gamma\left[1+\frac{\beta}{2}(j+1)\right]}
$$

and $J=\frac{(n-1)(\alpha+(n-2) \beta / 2)(\alpha n+\beta+(n-1) n \beta / 2)}{1+\alpha(n-1)+(n-2)(n-1) \beta / 2}$. These are the expressions used to make plots on Fig. 4.

Shot-noise, right edge: shifting all $T_{i} \rightarrow \frac{1}{2}+T_{i}$ gives $P_{p}^{(\beta)}(p)=\mathcal{N}_{\beta}^{-1} \int d[T] \Pi_{j}\left(\frac{1}{2}+T_{j}\right)^{\alpha-1}|\Delta(T)|^{\beta} \delta\left(\frac{n}{4}-p-\Sigma_{i} T_{i}^{2}\right)$, where the integration is now over the $n$-dimensional cube centered at origin: $-\frac{1}{2}<T_{i}<\frac{1}{2}$. Considering the right edge of the support of $P_{p}^{(\hat{\beta})}(p)$, thus $\frac{n}{4}-p \rightarrow 0$, one can then rescale $T_{i}$ $\rightarrow T_{i} \sqrt{\frac{n}{4}-p}$ and obtain

$$
P_{p}^{(\beta)}(p) \simeq R_{p}(n / 4-p)^{n / 2+n(n-1) \beta / 4-1} e^{-(n / 4-p) Y},
$$

where $\quad R_{p}=\left(2^{(1-\alpha) n} / \mathcal{N}_{\beta}\right) \int_{-\infty}^{\infty} \cdots \int_{-\infty}^{\infty} d[T]|\Delta(T)|^{\beta} \delta\left(1-\Sigma_{i} T_{i}^{2}\right)$. This integral can be further reduced to a Gaussian-type integral found in $\mathrm{Mehta}^{5}$ (see p. 354). After some algebra, we finally arrive at 


$$
R_{p}=\frac{\pi^{n / 2}}{2^{(\alpha-1) n+\beta(n-1) n / 4} \Gamma\left(r_{p}+1\right)} \prod_{j=0}^{n-1} \frac{\Gamma\left[1+\alpha+\frac{\beta}{2}(n+j-1)\right]}{\Gamma\left(1+\frac{\beta}{2} j\right) \Gamma\left(\alpha+\frac{\beta}{2} j\right)},
$$

where $r_{p}$ is given by Eq. (56). The correction factor $e^{-(n / 4-p) Y}$, with $Y=2(\alpha-1)-2(\alpha-1)^{2} /\left[1+\frac{\beta}{2}(n-1)\right]$, is found as before by expanding the rest in powers of $\frac{n}{4}-p$ and retaining the linear part in the exponent.

Shot-noise, left edge: for $p \rightarrow 0$, one gets positive contributions to $P_{p}^{(\beta)}(p)$ from all $2^{n}$ corners of the integration cube. As function $T(1-T)$ is not monotonous, it is more convenient to treat the contributions from all the corners separately. To this end, we make for $(n-m)$ variables $T_{k}$ the transformation $T_{k} \rightarrow\left(1-T_{k}\right)$ and then let all $T_{i}$ run from 0 to $\frac{1}{2}$. This gives

$$
\begin{aligned}
P_{p}^{(\beta)}(p)= & \frac{1}{\mathcal{N}_{\beta}} \sum_{m=0}^{n}\left(\begin{array}{c}
n \\
m
\end{array}\right) \int_{0}^{1 / 2} d[T] \prod_{i=1}^{m} \prod_{k=m+1}^{n} T_{i}^{\alpha-1}\left(1-T_{k}\right)^{\alpha-1} \mid 1 \\
& -T_{i}-\left.T_{k}\right|^{\beta} \prod_{1 \leq i<j \leq m}\left|T_{i}-T_{j}\right|^{\beta} \prod_{m+1 \leq k<l \leq n}\left|T_{k}-T_{l}\right|^{\beta} \delta(p \\
& \left.-\sum_{i} T_{i}\left(1-T_{i}\right)\right) .
\end{aligned}
$$

The factor $\left(\begin{array}{l}n \\ m\end{array}\right)$ appears since one has $\left(\begin{array}{l}n \\ m\end{array}\right)$ equivalent corners, and $2^{n}=\sum_{m=0}^{n}\left(\begin{array}{c}n \\ m\end{array}\right)$. Scaling $T_{i} \rightarrow p T_{i}$ yields

$$
P_{p}^{(\beta)}(p) \simeq \sum_{m=0}^{n}\left(\begin{array}{l}
n \\
m
\end{array}\right) Q(m) p^{\gamma(m)-1} \mathrm{e}^{p E(m)},
$$

with $\gamma(m)=n+m(\alpha-1)+\frac{\beta}{2}[m(m-1)+(n-m)(n-m-1)]$ and

$$
\begin{gathered}
Q(m)=\frac{1}{\Gamma[\gamma(m)]} \prod_{j=0}^{n-1} \frac{\Gamma\left[1+\alpha+\frac{\beta}{2}(n+j-1)\right]}{\Gamma\left(\alpha+j \frac{\beta}{2}\right)} \prod_{j=0}^{m-1} \frac{\Gamma\left[\alpha+j \frac{\beta}{2}\right] \Gamma\left[1+\frac{\beta}{2}(j+1)\right]}{\Gamma\left[1+\frac{\beta}{2}(n-m+j)\right] \Gamma\left[1+\frac{\beta}{2}(n-m+j+1)\right]}, \\
E(m)=3-\alpha+\beta(n-2 m-1)+\frac{2 m}{\gamma(m)}[\alpha-1+\beta(2 m-n)]\left[\alpha+\frac{\beta}{2}(m-1)\right] .
\end{gathered}
$$

For the given even $n=2 k$ or odd $n=2 k+1, k \geq 1$, the function $\gamma(m)$ has a minimum at $m=k$ that gives the leading exponent $\ell_{p}=\gamma(k)-1$ stated in Eq. (56), and $L_{p}=\left(\begin{array}{l}n \\ k\end{array}\right) Q(k)$.

${ }^{1}$ C. W. J. Beenakker, Rev. Mod. Phys. 69, 731 (1997).

${ }^{2}$ Ya. M. Blanter and M. Büttiker, Phys. Rep. 336, 1 (2000).

${ }^{3}$ R. Landauer, IBM J. Res. Dev. 1, 223 (1957); V. A. Khlus, Sov. Phys. JETP 66, 1243 (1987); G. B. Lesovik, JETP Lett. 49, 592 (1987); M. Büttiker, Phys. Rev. Lett. 65, 2901 (1990).

${ }^{4}$ Y. Alhassid, Rev. Mod. Phys. 72, 895 (2000).

${ }^{5}$ M. L. Mehta, Random Matrices, 2nd ed. (Academic Press, New York, 1991).

${ }^{6}$ H. U. Baranger and P. A. Mello, Phys. Rev. Lett. 73, 142 (1994).

${ }^{7}$ R. A. Jalabert, J.-L. Pichard, and C. W. J. Beenakker, EPL 27, 255 (1994).

${ }^{8}$ P. J. Forrester and S. O. Warnaar, Bull. Am. Math. Soc. 45, 489 (2008).

${ }^{9}$ D. V. Savin and H.-J. Sommers, Phys. Rev. B 73, 081307(R) (2006).

${ }^{10}$ M. Novaes, Phys. Rev. B 75, 073304 (2007).

${ }^{11}$ H.-J. Sommers, W. Wieczorek, and D. V. Savin, Acta Phys. Pol. A 112, 691 (2007).

${ }^{12}$ D. V. Savin, H.-J. Sommers, and W. Wieczorek, Phys. Rev. B 77, 125332 (2008).

${ }^{13}$ P. W. Brouwer and C. W. J. Beenakker, J. Math. Phys. 37, 4904 (1996).

${ }^{14}$ J. E. F. Araújo and A. M. S. Macêdo, Phys. Rev. B 58, R13379 (1998).

${ }^{15}$ P. A. Mello and H. U. Baranger, Waves Random Media 9, 105 (1999).
${ }^{16}$ Ya. M. Blanter, H. Schomerus, and C. W. J. Beenakker, Physica E (Amsterdam) 11, 1 (2001).

${ }^{17}$ O. M. Bulashenko, J. Stat. Mech.: Theory Exp. 2005, P08013.

${ }^{18}$ E. N. Bulgakov, V. A. Gopar, P. A. Mello, and I. Rotter, Phys. Rev. B 73, 155302 (2006).

${ }^{19}$ V. A. Gopar, S. Rotter, and H. Schomerus, Phys. Rev. B 73, 165308 (2006).

${ }^{20}$ B. Béri and J. Cserti, Phys. Rev. B 75, 041308(R) (2007).

${ }^{21}$ P. Vivo and E. Vivo, J. Phys. A 41, 122004 (2008).

${ }^{22}$ K. Richter and M. Sieber, Phys. Rev. Lett. 89, 206801 (2002).

${ }^{23}$ P. Braun, S. Heusler, S. Müller, and F. Haake, J. Phys. A 39, L159 (2006).

${ }^{24}$ S. Müller, S. Heusler, P. Braun, and F. Haake, New J. Phys. 9, 12 (2007).

${ }^{25}$ M. Novaes, Phys. Rev. B 78, 035337 (2008).

${ }^{26}$ V. A. Osipov and E. Kanzieper, Phys. Rev. Lett. 101, 176804 (2008).

${ }^{27}$ V. A. Osipov and E. Kanzieper, arXiv:0902.3069 (unpublished).

${ }^{28}$ A. García-Martín and J. J. Sáenz, Phys. Rev. Lett. 87, 116603 (2001).

${ }^{29}$ M. H. Pedersen, S. A. van Langen, and M. Büttiker, Phys. Rev. B 57, 1838 (1998).

${ }^{30}$ P. Vivo, S. N. Majumdar, and O. Bohigas, Phys. Rev. Lett. 101, 216809 (2008).

${ }^{31}$ P. W. Brouwer and C. W. J. Beenakker, Phys. Rev. B 55, 4695 (1997). 
${ }^{32}$ A. G. Huibers, S. R. Patel, C. M. Marcus, P. W. Brouwer, C. I. Duruöz, and J. S. Harris, Phys. Rev. Lett. 81, 1917 (1998).

${ }^{33}$ S. Hemmady, J. Hart, X. Zheng, T. M. Antonsen, E. Ott, and S. M. Anlage, Phys. Rev. B 74, 195326 (2006).

${ }^{34}$ S. Oberholzer, E. V. Sukhorukov, C. Strunk, C. Schonenberger, T. Heinzel, and M. Holland, Phys. Rev. Lett. 86, 2114 (2001); S. Oberholzer, E. V. Sukhorukov, and C. Schönenberger, Nature (London) 415, 765 (2002).

${ }^{35}$ S. Gustavsson, R. Leturcq, M. Studer, I. Shorubalko, T. Ihn, K. Ensslin, D. C. Driscoll, and A. C. Gossard, Surf. Sci. Rep. 64, 191 (2009).

${ }^{36}$ L. K. Hua, Harmonic Analysis of Functions of Several Complex Variables in the Classical Domains (American Mathematical Society, Providence, RI, 1963).

${ }^{37}$ I. G. MacDonald, Symmetric Functions and Hall Polynomials (Oxford University Press, Oxford, 1998).

${ }^{38}$ This follows from the Jacobi-Trudi identity expressing the Schur functions through elementary symmetric functions, the latter being polynomials in matrix entries when evaluated at the eigenvalues.

${ }^{39}$ Y. V. Fyodorov and B. A. Khoruzhenko, J. Phys. A 40, 669 (2007).

${ }^{40}$ For example, see problem 68 in Part Two in the textbook by G. Pólya and G. Szegö, Problems and Theorems in Analysis. Volume I: Series, Integral Calculus, Theory of Functions (Springer, New York, 1976).

${ }^{41}$ A. B. Balantekin, Phys. Rev. D 62, 085017 (2000).

${ }^{42}$ K. W. J. Kadell, Adv. Math. 130, 33 (1997).
${ }^{43}$ J. Kaneko, SIAM J. Math. Anal. 24, 1086 (1993).

${ }^{44}$ Z. M. Yan, Can. J. Math. 44, 1317 (1992).

${ }^{45}$ In the mathematical literature the inverse of $c_{\lambda}$ is known as the product of all hook lengths of the partition $\lambda$.

${ }^{46}$ The structure of Eq. (18b) has been verified by symbolical computation of Eq. (17) in Mathematica, however, its exact analytical proof is still lacking. Our analysis suggests that for those partitions which have all the $a_{j}$ 's distinct, the corresponding $b_{j}$ 's are given by $b_{j}=a_{j}-\lambda_{j}(2 / \beta-1)$. In the general case, finding similar relation between these two objects is an interesting open problem.

${ }^{47}$ B. L. Altshuler, V. E. Kravtsov, and I. V. Lerner, Zh. Eksp. Teor. Fiz. 91, 2276 (1986) [Sov. Phys. JETP 64, 1352 (1986)].

${ }^{48}$ H. D. Politzer, Phys. Rev. B 40, 11917 (1989).

${ }^{49}$ C. W. J. Beenakker, Phys. Rev. Lett. 70, 1155 (1993).

${ }^{50}$ Pfaffian, or quaternion determinant, of an antisymmetric matrix $A$ is defined as follows: $(\operatorname{Ref} .5) \operatorname{Pfaff}(A)=\sqrt{\operatorname{det}(A)}$. It is important to note that a Pfaffian is analytic in the matrix entries. Also, in the particular case of unitary symmetry, $\beta=2$, one generally has $A=i \sigma_{2} \otimes \tilde{A}$, with $\sigma_{2}$ being the corresponding Pauli matrix in the quaternion subspace, thus $\operatorname{Pfaff}(A) \equiv \operatorname{det}(\widetilde{A})$ in this case.

${ }^{51}$ H. Cramér, Mathematical Methods of Statistics (Princeton University Press, Princeton, 1957).

${ }^{52}$ S. Blinnikov and R. Moessner, Astron. Astrophys. Suppl. Ser. 130, 193 (1998).

${ }^{53}$ H.-J. Sommers and W. Wieczorek, J. Phys. A 41, 405003 (2008). 